\title{
Linfoma de Hodgkin con afectación ósea: comunicación de un caso y revisión de la literatura
}

\author{
D. Márquez Medina*, B. Márquez Lobo**, M. C. Talavera Hernández***, I. Blancas López-Barajas*, \\ I. Sáez Medina****, J. L. García Puche***
}

\begin{abstract}
Resumen
Existen pocas comunicaciones en la literatura acerca de la afectación ósea primaria por linfoma de Hodgkin. El linfoma de Hodgkin representa menos del $0^{\prime} 15 \%$ de los tumores malignos primarios del hueso, aunque por radiología es frecuente detectar lesiones óseas en pacientes con enfermedad de Hodgkin. Comunicamos el caso de un paciente inicialmente diagnosticado de linfoma de Hodgkin en fémur izquierdo, tratado con un régimen combinado de quimioradioterapia secuencial, que permanece libre de recaída a cuatro años después, aprovechando para revisar la literatura respecto a su diagnóstico y tratamiento.
\end{abstract}

Palabras clave:

Afectación ósea. Linfoma de Hodgkin. Quimioterapia. Radioterapia. Tratamiento.

Oncología, 2004; 27 (3):135-139

* F.E.A. de Oncología Médica

** M.I.R. de Anatomía Patológica

*** F.E.A. de Oncología Médica y Radioterápica. Jefes de Sección de Oncología Médica y RT

**** M.I.R. de Oncología Médica

Unidad integral de Oncología y Servicio de Anatomía Patológica. Hospital San Cecilio. Granada (España) 


\title{
D. Márquez Medina y cols.
}

\section{Summary}

\begin{abstract}
There are few literature reports about bone primary Hodgkin's lymphoma. Hodgkin's lymphoma represents less than $0.15 \%$ of the primary malignant bone tumors, although radiology studies frequently detect bone lesions in patients with Hodgkin's disease. We report the case of a patient that was initially diagnosed of bone Hodgkin's lymphoma with left femur involvement. The patient was treated by a combined regime of sequential chemo-radiotherapy in our Oncology Unit. He remains relapse-free three years after diagnosis. Taking advantage of our report we reviewed the literature looking for diagnostic and treatment strategies for the process.
\end{abstract}

Key words: Hodgkin's lymphoma. Osseous involvement. Chemotherapy. Radiotherapy.

\section{Introducción}

Los linfomas óseos son infrecuentes' (del 3 al $8 \%$ de los cánceres primarios del hueso) y principalmente linfomas no Hodgkin ( $\mathrm{LNH}$ ) de células $\mathrm{B}^{2}$. El linfoma de Hodgkin (LH) sólo representa un 3\% de los linfomas óseos, lo cual explica la escasez de casos comunicados en la literatura.

Los LH generalmente se presentan como linfoadenopatías que se extienden ordenadamente, siendo frecuente la afectación de la médula ósea, sin destrucción del hueso, en las etapas avanzadas de la enfermedad (5-15\%). A diferencia de ella, la afectación ósea sintomática por LH destruye el tejido y es una forma inusual de debut en esta población, aunque por radiología se puedan detectar lesiones óseas en el $10-20 \%$ de los pacientes con $\mathrm{LH}^{3}$.

Se debe distinguir entre a) $\mathrm{LH}$ primario óseo (12$15 \%$ ) donde el hueso es la única localización afectada; b) Afectación ósea inicial por $\mathrm{LH}(\mathrm{AOLH})$, la más común, donde es frecuente encontrar adenopatías simultáneas, y c) Lesiones óseas en el curso de un $\mathrm{LH}$.

La AOLH generalmente se presenta entre la segunda y quinta década de la vida, aunque ha sido descrita a cualquier edad, con mayor riesgo de padecerla pacientes varones (1'4: 1) con un máxima incidencia hacia los 31 años (rango de 5 a 83 años).

A excepción de aquellas lesiones óseas que aparecen en la evolución de un $\mathrm{LH}$, el diagnóstico de la AOLH es siempre difícil. La AOLH suele presentarse con dolor como único síntoma y se confunde otras entidades $^{4}$ (osteomielitis, artritis o granuloma eosinófilo), con lo que su diagnóstico definitivo se retrasa durante meses ${ }^{5}$ incluso en aquellos casos asociados a adenopatías, donde los síntomas B aparecen en más del $50 \%$ de los pacientes.

No existen diferencias radiológicas entre la $\mathrm{AOLH}$ inicial y las lesiones recurrentes. Ambas incluyen osteolisis, esclerosis y lesiones mixtas que pueden mostrar re- acción perióstica y muy diversa distribución. Ostrowski ${ }^{1}$ comunica un $26 \%$ de lesiones líticas, $26 \%$ escleróticas y $43 \%$ mixtas, Newcomer ${ }^{3} 24 \%, 20 \%$ y $15 \%$ y Granger 6 $75 \%, 15 \%$ y $5 \%$ respectivamente. Reacciones periósticas, destrucción cortical y masas de tejidos blandos pueden observarse en más del $50 \%$, pero las fracturas patológicas son raras. Esto contrasta con la imagen típicamente permeativa de la afectación ósea por $\mathrm{LNH}$.

Aunque las modernas técnicas de imagen como la TAC, la resonancia nuclear magnética o la gammagrafía con galio ${ }^{6,7}$ complementan a la radiología convencional 7 , todas las lesiones deberían ser confirmadas con biopsia ósea y ganglionar simultaneas.

Las áreas más comúnmente afectadas en $\mathrm{AOLH}$ son pelvis, columna y fémur, pero también se han descrito en esternón, escápula, clavícula, costillas, cráneo y ti$\mathrm{bia}^{1}$. Las lesiones solitarias son tan frecuentes como las múltiples ${ }^{3}$.

El diagnóstico anatomopatológico de $\mathrm{AOLH}$ es tan complicado como el clínico. Antes del desarrollo de las modernas técnicas de inmunohistoquímica, los errores diagnósticos eran frecuentes, especialmente con linfomas anaplásicos y de células T. Por tanto, la confirmación inmunohistoquímica debe ser obligada en el diagnóstico del LH extranodal. Una biopsia de linfoadenopatías que aparezcan sincrónicamente puede ser de utilidad, dada la escasa rentabilidad de la citología e histología ósea.

Las láminas de hueso son preparadas generalmente en formalina al $10 \%$ o parafina teñida con hematoxilina-eosina y la AOLH generalmente se presenta como un infiltrado de linfocitos, células plasmáticas, eosinófilos, histiocitos y células mononucleares atípicas entre trabéculas intactas'. Estas imágenes son demasiado similares a las de los LNH óseos, donde células similares a las de Reed-Stemberg pueden aparecer. También puede aparecer necrosis, fibrosis e inflamación y, ocasionalmente, áreas reactivas de osteoformación en los casos con es- 
clerosis y reacción periostal radiológica. La fibrosis y la esclerosis pueden ser una forma predominante en la afectación ósea por $\mathrm{LH}$ tipo esclerosis nodular.

Para inmunohistología, usualmente se utilizan anticuerpos contra queratinas, CAM5.2, S-100, vimentina, desmina, CD-1, 3, 15, 22, 30, 43, 45, 45Ro y L-26. Suele existir positividad a CD-15 y 30 , sin reactividad a marcadores $B \circ T$. Positividad a CD-45 y reagrupamientos en los genes de las inmunoglobulinas o receptores TcR no son muy usuales. De cualquier modo, los distintos subtipos de LH podrían mostrar diferentes inmunofenotipos $^{2}$ : La esclerosis nodular, celularidad mixta y depleción linfocítica expresan CD-15 y 30, sin CD45 o marcadores $B$, que pueden aparecer en la variante de predominio linfocítico. La esclerosis nodular y la celularidad mixta son los tipos más frecuentes de $\mathrm{AOLH}^{3}$. Las otras variantes son excepcionales.

Distintas estrategias que combinan quimioterapia (QT), radioterapia (RT) y cirugía han sido empleadas en el tratamiento de la AOLH. Los escasos casos de LH óseo primario han sido tratados con RT o resección, consiguiendo largas supervivencias incluso en aquellos con afectación multifocal'. La QT obtiene buenas tasas de respuesta en $\mathrm{LH}$ óseo primario y $\mathrm{AOLH}$ presente simultáneamente en hueso y fuera de él.

Pueden utilizarse pautas de QT para LH y LNH. Esquemas comprobados y eficaces son MOPP (mostaza nitrogenada, vincristina, procarbazina y prednisona), ABVD (adriamicina, bleomicina, vinblastina, dacarbazina) y EVA (etoposido, vincristina, doxorubicina). La combinación MOPP/ABVD por 6-8 ciclos alternados con consolidación RT sobre el hueso afecto ha sido descrito en casos clínicos publicados como una terapia eficaz en seguimentos de 1-16 años. Se emplean dosis de radiación de 30 a $40 \mathrm{~Gy}$, con fotones de $6 \mathrm{MeV}$ de aceleradores lineales o fotones de 1'25 MeV de telecobaltoterapia ${ }^{8}$.

La modalidad de tratamiento combinado QT y RT ha demostrado una gran tasa de respuesta y de control a largo tiempo, con supervivencias del 60 al $84 \%$ en nueve años. Se han descrito fallos en la inducción de la respuesta, pero escasas recaídas tras la terapia combinada. En las recurrencias, RT o combinaciones de QT y RT obtienen supervivencias de meses a años, incluso la curación del proceso.

La demora en el diagnóstico, la refractariedad al tratamiento o la recaída implican un peor pronóstico pero, al contrario de los pacientes con afectación de la médula ósea (estadio IV), la AOLH (estadio IE-IIIE) no parece conllevar un peor pronóstico o respuesta que los casos con exclusiva afectación ganglionar e igual estadio (estadio I-III)2. Ambos presentan la misma evolución al tratamiento ${ }^{5}$.

\section{Caso clínico}

Presentamos el caso de un paciente varón de 50 años con crisis de sudoración profusa, pérdida del $10 \%$ del peso corporal y dolor en rodilla izquierda, sin fiebre, de seis meses de evolución, que consulta por dolor y aumento de tamaño en el muslo izquierdo. En su analítica (incluyendo LDH) no se apreciaron valores anormales y, clínicamente, fue diagnosticado de osteomielitis del trocánter izquierdo. La radiología de pelvis mostró una única lesión lítica en la cabeza femoral izquierda confirmada por TAC, ${ }^{99} \mathrm{Tc}$ y ${ }^{67} \mathrm{Ga}$ gammagrafía. La biopsia ósea fue informada como afectación linfoproliferativa ósea con posibilidad de tratarse de un $\mathrm{LH}$.

Un mes después, remitido a nuestra Unidad de Oncología, el examen físico reveló una masa adenopática de $7 \mathrm{cms}$ de diámetro en la axila izquierda y adenopatías bilaterales inguinales de meses de evolución. La sintomatología previa fue interpretada como síntomas $B$ y nuevos datos de laboratorio mostraban una $\beta_{2} \mu$ globulina de $5 \mathrm{mg} / \mathrm{l}$, LDH 670 y serología de $\mathrm{VIH}$ y hepatovirus negativa.

Se realizó biopsia de la masa axilar, informada como LH tipo esclerosis nodular. La arquitectura del ganglio estaba distorsionada por tractos fibrosos entre nódulos de linfocitos, células plasmáticas, neutrófilos, histiocitos y células CD-30 +, con citoplasma claro y núcleos lobulados. Existía una positividad focal para CD15 y EMA y casi todas las células tumorales eran $\mathrm{Ki}$ 67 +. La biopsia de médula ósea fue informada como fibrosis asociada a infiltración por LH. La TAC mostraba adenopatías en la axila izquierda, supraclaviculares, mediastino, retroperitoneo, pelvis e ingles.

Del 24-2-2000 al 29-9-2000, el paciente recibió siete ciclos de $Q T$ en régimen $A B V D$. Al segundo ciclo, los síntomas $B$ y las adenopatías periféricas habían desaparecido. Al cuarto, los valores de $\beta_{2} \mu$ globulina (1'7 mg/l) y LDH (398) eran normales, y, al sexto, la TAC sólo mostraba esclerosis de la pala ilíaca izquierda, la biopsia de médula ósea no presentaba infiltración por LH y la gammagrafía con ${ }^{67}$ Galio fue negativa. El 15-1-2001, se consolidó la respuesta con 3 ciclos de QT en régimen MOPP y con RT (36 Gy) sobre el fémur izquierdo. Hasta el día de hoy el paciente se encuentra libre de recaída.

\section{Regímenes de QT empleados en el tratamiento}

ABVD (cada 2 semanas): Doxorubicina $25 \mathrm{mg} / \mathrm{m}^{2}$ + Bleomicina $10 \mathrm{mg} / \mathrm{m}^{2}$ + Vinblastina $6 \mathrm{mg} / \mathrm{m}^{2}+\mathrm{Da}$ carbazina $375 \mathrm{mg} / \mathrm{m}^{2}$.

COPP (cada 4 semanas): Día 1: Ciclofosfamida 650 $\mathrm{mg} / \mathrm{m}^{2}+$ Vincristina $1^{\prime} 4 \mathrm{mg} / \mathrm{m}^{2}+$ Procarbazina 100 


\section{Márquez Medina y cols.}

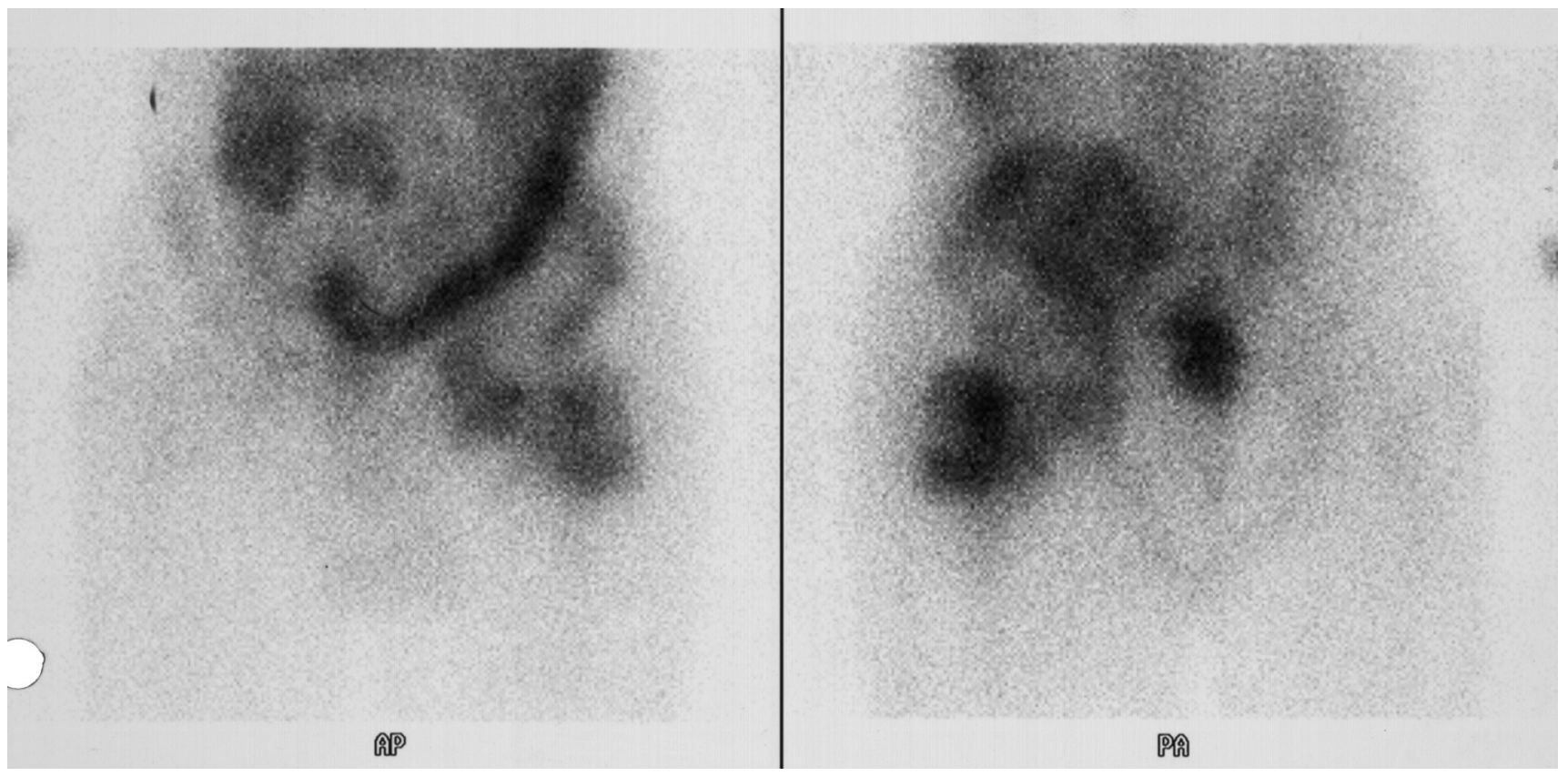

Fig. 1. Gammagrafía con Galio ${ }^{67}$ inicial del paciente, apreciándose captación patológica en cabeza femoral izquierda.

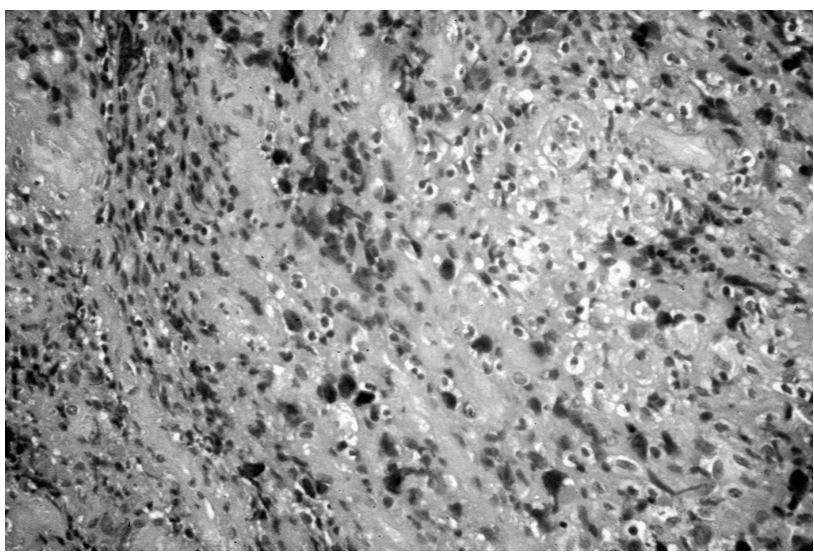

Fig. 2. Biopsia de hueso mostrando células atípicas mononucleares de escaso citoplasma, fuertemente eosinófilas, sueltas y en acúmulos, en un estroma de tejido fibroso, acompañadas por una población de células reactivas compuestas por neutrófilos, eosinófilos, linfocitos e histiocitos. Las células tumorales expresan cd30 y cd1 5 .

$\mathrm{mg} / \mathrm{m}^{2}$. Día 8: Ciclofosfamida $650 \mathrm{mg} / \mathrm{m}^{2}+$ Vincristina 1'4 mg/m². Días 1-14: Prednisona $40 \mathrm{mg} / \mathrm{m}^{2}$.

\section{Discusión}

Dos motivos nos han inducido a comunicar este caso. La escasa incidencia de LH óseos. La afectación de médula ósea puede ser un hallazgo usual en etapas avanzadas de la enfermedad, pero la AOLH representa sólo un $0^{\prime} 15 \%$ de los tumores malignos del hueso. Existen únicamente unos 30 casos publicados en la literatura, y éste es el primero diagnosticado y tratado en nuestro centro en los últimos 20 años.

El segundo motivo es pensar que un mayor conocimiento de la clínica, diagnóstico y terapéutica de la AOLH puede contribuir a un mejor manejo de la enfermedad. La escasa incidencia de linfomas en el hueso y la aún menor incidencia de la AOLH explica gran parte de las demoras y confusiones en su diagnóstico. La ausencia de síntomas o hallazgos radiológicos específicos hace el resto. Por tanto, recordar la posibilidad de que exista esta dolencia en una primera evaluación del paciente ayudará al especialista a reducir la dificultad y retraso de su diagnóstico.

La realización de una historia clínica completa con una exploración física sistemática también es importante. La forma de presentación más frecuente de la AOLH va acompañada de adenopatías simultáneas. Nuestro paciente refería dolor, síntomas B evidentes y múltiples masas adenopáticas meses antes de ser diagnosticado de osteomielitis. Afortunadamente, su biopsia de hueso condujo directamente al diagnóstico de $\mathrm{AOLH}$, pero ampliar la exploración o el interrogatorio podrían simplificar bastante el diagnóstico en otros muchos casos. De hecho, la precocidad de éste es especialmente importante si consideramos que la $\mathrm{AOLH}$ 
no empeora inicialmente el pronóstico de la enfermedad más de la afectación ganglionar pero, por el contrario, la progresión a un estadio superior del proceso Igeneralmente asociado a evoluciones más prolongadas) se acompaña de menores tasas de curación y de recaídas más frecuentes. Así la $\mathrm{AOLH}$ debe tratarse lo antes posible para promover la supervivencia del paciente.

La clínica y presentación de nuestro caso fue similar a las descritas en la literatura: Afectación ósea y ganglionar sincrónica en un paciente varón adulto. La lesión ósea era única (que son tan comunes como las múltiples) y lítica (24 al $75 \%$ de los casos) en la cabeza femoral izquierda (una de la localizaciones más frecuentes de la AOLH).

Diversos autores han relacionados los hallazgos clínicos y radiológicos de la $\mathrm{AOLH}$ con las diferentes formas de diseminación del $\mathrm{LH}^{9}$, considerando que las lesiones líticas se deben a una diseminación hematógena y que una extensión directa al hueso desde ganglios adyacentes origina las escleróticas. Para otros autores, es la afectación ósea multifocal la que sostiene la hipótesis de una diseminación hematógena y la común afectación del esqueleto axial la de una extensión directa desde los ganglios vecinos ${ }^{5}$. También se ha descrito una mayor incidencia de AOLH multifocal en pacientes con afectación ósea inicial y $\mathrm{LH}$ de la variedad celularidad mixta $^{3}$, pero este aspecto no ha sido confirmado.

Otro hecho a discutir es la dificultad del diagnóstico histológico. La AOLH puede confundirse fácilmente con otras entidades y la biopsia suele rendir poca información. Por tanto, conviene realizar un análisis de adenopatías simultáneas y confirmar los hallazgos con técnicas de inmunohistoquímica.

Se pueden emplear diferentes estrategias terapéuticas en la AOLH pero, como hemos descrito, la afectación ósea no empeora el pronóstico del $\mathrm{LH}$ y puede responder a QT y RT. Por tanto, la cirugía debería reservarse para la obtención de muestras, resolver complicaciones (usualmente escasas) y para aquellos casos sin respuesta al tratamiento.

Nuestro paciente fue tratado con un esquema de QT similar a los descritos en la bibliografía. Ciclos combinados y alternantes de MOPP y ABVD se reemplazaron por un esquema secuencial de ABVD (7 ciclos), consolidado con COPP (3 ciclos) y RT, dada la extensión de la enfermedad (estadio-IV). Preferimos relegar la consolidación de la afectación del hueso hasta haber conseguido una completa respuesta en médula ósea, que era la que realmente definía el pronóstico del paciente. Las dosis y cronología de la QT y de la RT fueron similares a las que aparecen en la literatura.
Tras casi cuatro años, el paciente permanece sin evidencia de enfermedad y concluimos que la $\mathrm{AOLH}$ es una entidad poco frecuente que responde al tratamiento, cuyo diagnóstico precoz debe buscarse para mejorar el pronóstico de los enfermos.

\section{Bibliografía}

1. Ostrowski ML, Inwards CY, Strickler JG, Witzig TE, Wenger DE, Unni KK. Osseous Hodgkin disease. Cancer 1999; 85: $1166-78$.

2. Ozdemirli M, Mankin HJ, Aisenberg AC, Harris NL. Hodgkin's disease presenting as a solitary bone tumor. A report of four cases and review of the literature. Cancer 1996; 77: 79-88.

3. Newcomer LN, Silverstein MB, Cadman EC, Farber LR, Bertino JR, Prosnitz LR. Bony involvement in Hodgkin's disease. Cancer 1982; 49: 338-42.

4. Cowie F, Benghiat A, Holgate C. Primary Hodgkin's disease of bone. Clin Oncol (R Coll Radiol) 1991; 3: 233-5.

5. Jaubert D, Eghbali $H$, Fuzibet JG, Pesce A, Hovette $P$, Delmarre B, Hauteville D, Hoerni B. [Bone localizations disclosing Hodgkin's disease. Apropos of 11 cases]. Rev Med Interne 1992; 13: 21-6.

6. Granger W, Whitaker R. Hodgkin's disease in bone, with special reference to periosteal reaction. Br J Radiol 1967; 40: 939-48.

7. Ferrant A, Rodhain J, Michaux JL, Piret L, Maldague B, Sokal G. Detection of skeletal involvement in Hodgkin's disease: a comparison of radiography, bone scanning and bone marrow biopsy in 38 patients. Cancer 1975; 35: 1346-53.

8. Borg MF, Chowdhury AD, Bhoopal S, Benjamin CS. Bony involvement in Hodgkin's disease. Australas Radiol 1993; 37: 63-6.

9. Edeiken-Monroe B, Edeiken J, Kim EE. Radiologic concepts of lymphoma of the bone. Radiol Clin North Am 1990; 28: 841-64.

Correspondencia:

Dr. D. Márquez Medina

Secretaría de Servicio de Oncología Médica y Radioterápica Hospital Clínico Universitario San Cecilio de Granada

Avda. Doctor Olóriz, 16

E-18012 Granada

E-mail:dmarmed@hotmail.com 